\title{
Dual Spacecraft Observations of Lobe Magnetic Field Perturbations Before, During and After Plasmoid Release
}

\author{
J.A. Slavin ${ }^{1}$, M. Hesse ${ }^{1}$, C.J. Owen ${ }^{2}$, S. Taguchi ${ }^{3}$, D.H. Fairfield ${ }^{1}$, R.P. Lepping ${ }^{1}$, \\ S. Kokubun ${ }^{4}$, T. Mukai ${ }^{5}$, A.T.Y. Lui ${ }^{6}$, R.R. Anderson ${ }^{7}$, H. Matsumoto ${ }^{8}$ and \\ P.R. Sutcliffe ${ }^{9}$
}

\begin{abstract}
This study examines a data set returned by IMP 8 and Geotail on January 29, 1995 during a substorm which resulted in the ejection of a plasmoid. The two spacecraft $(\mathrm{s} / \mathrm{c})$ were situated in the north lobe of the tail and both observed a traveling compression region (TCR). We show that in this instance dual s/c measurements can be used to model all three dimensions of the underlying plasmoid and to estimate its rate of expansion. For this event plasmoid dimensions of $\Delta \mathrm{X} \sim 18, \Delta \mathrm{Y} \sim 30$, and $\Delta \mathrm{Z} \sim$ $10 \mathrm{R}_{\mathrm{e}}$ are determined from the IMP 8 and Geotail observations. Furthermore, a factor of $\sim 2$ increase in the amplitude of the TCR occurred in the $1.5 \mathrm{~min}$ it took to move from IMP 8 to Geotail. Modeled using conservation of magnetic flux, this increase in lobe compression implies that the underlying plasmoid was expanding at a rate of $\sim 140 \mathrm{~km} / \mathrm{s}$. Finally, a reconfiguration of the lobe magnetic field followed plasmoid ejection which moved magnetic flux tubes into the wake behind the plasmoid where they would become available to feed the reconnection region.
\end{abstract}

\section{Introduction}

Traveling compression regions are several minute long enhancements of the magnetic field in the lobes of the tail during which there is a north-then-south variation in $B_{Z}$ relative to a mean baseline (Slavin et al., 1993). The greater north-south dimensions of the plasmoid relative to the plasma sheet result in the lobe field lines being compressed and draped about the plasmoid as it moves down the tail. This local compression of the lobe magnetic field then accompanies the plasmoid as it moves down the tail and can be observed by spacecraft residing in the lobes. In this paper we report the first dual spacecraft observations of lobe magnetic field draping and compression before, during, and after the formation and ejection of a plasmoid. The results provide new information regarding

\footnotetext{
${ }^{1}$ Laboratory for Extraterrestrial Physics, NASA/GSFC, Greenbelt, MD

${ }^{2}$ Astronomy Unit, Queen Mary and Westfield College, London, U.K.

${ }^{3}$ Dept. of Electronic Engineering, The University of

Electro-Communications, Tokyo, Japan

${ }^{4}$ Solar-Terrestrial Environment Laboratory, Nagoya University., Japan

${ }_{6}^{5}$ Institute of Space and Astronautical Science, Kanagawa, Japan

6 JHU/Applied Physics Laboratory, Johns Hopkins Road, Laurel, MD

7 Department of Physics and Astronomy, University of Iowa, lowa City

${ }^{8}$ Radio Atmospheric Science Center, Kyoto Unıversity, Gokanosho, Japan

'Hermanus Magnetic Observatory, South Africa
}

Copyright 1999 by the American Geophysical Union. plasmoid dimensions, their evolution and the lobe reconfiguration immediately following plasmoid.

\section{IMP 8 and Geotail TCRs}

On January 29, 1995 IMP 8 and Geotail were both located in the tail. An episode of strong tail loading/unloading began around 19:40 UT with the lobe field intensity at $\sim 23 \mathrm{nT}$ and $\mathrm{B}_{\mathbf{z}}$ $\sim 0$ as measured by IMP 8 . The lobe field strength then increased by $-10 \mathrm{nT}$ over the next hour as the flaring of the field grew until $B_{z} \sim-7 n T$. This tail loading event was well correlated with a southward turning of the IMF at WIND which was followed by variable, largely southward IMP $B_{z}$ until about 21:00. A TCR was detected by IMP 8 at 21:13 near the peak in this tail loading. The lobe field was then observed to "unload" over the next $\sim 50$ min back to B-total $\sim 25 \mathrm{nT}$ and $\mathrm{B}_{\mathrm{z}} \sim 0$.

Figure 1 displays IMP 8 and Geotail measurements for 21:00 $-21: 30$ during the peak of the loading interval. The onset of a substorm is evident at 21:09 on the basis of AKR emissions detected by Geotail and $\mathrm{Pi} 2$ pulsations at Hermanus, South Africa. About 2 and 5 minutes later, respectively, a negative magnetic bay at the IMAGE chain (Pello) and an energetic electron enhancement at LANL S/C 1987-097 (local time 4:06) were observed (not shown). We attribute these later observations of high latitude negative bays in Scandinavia and energetic electrons at S/C 1987-097 to propagation delays associated with the locations of the observing stations.

TCRs in the IMP 8 database have recently been analyzed in detail by Taguchi et al. (1998). Their results are of special interest because at distances of $X \sim-26$ to $-38 R_{e}$, the region sampled by IMP 8 , the TCR signature is usually somewhat modified from that seen in the more distant tail. In particular, only a portion of the complete signature of field line draping over the plasmoid is usually observed. The reason is that plasmoids initially form in this region with part of the plasmoid lying earthward of IMP 8 and part tailward. In contrast, a s/c located much further down the tail will observe the complete $\pm B_{z}$ perturbation as the plasmoid passes.

The TCR observed by IMP 8 on Jan 29, 1995 has many of the characteristics described above. The compression began about 1-2 min after the substorm onset at 21:09 and peaked at 21:12:50. The presence of only a southward shift in $B_{z}$ starting $\sim 1 \mathrm{~min}$ before the peak compression in B-total is consistent with the plasmoid forming underneath IMP 8 and then beginning to move down the tail while it was still growing. The duration of the TCR based upon the compression was $\sim 2 \mathrm{~min}$ with an amplitude of $\Delta \mathrm{B} / \mathrm{B} \sim 6 \%$. No $\mathrm{B}_{\mathrm{y}}$ signature is present. This is indicative of the $s / c$ either passing over the middle of $a$ centralized plasmoid bulge or simply a bulge extending to large distances in the $\pm Y$ (e.g., see Slavin et al., 1993). 


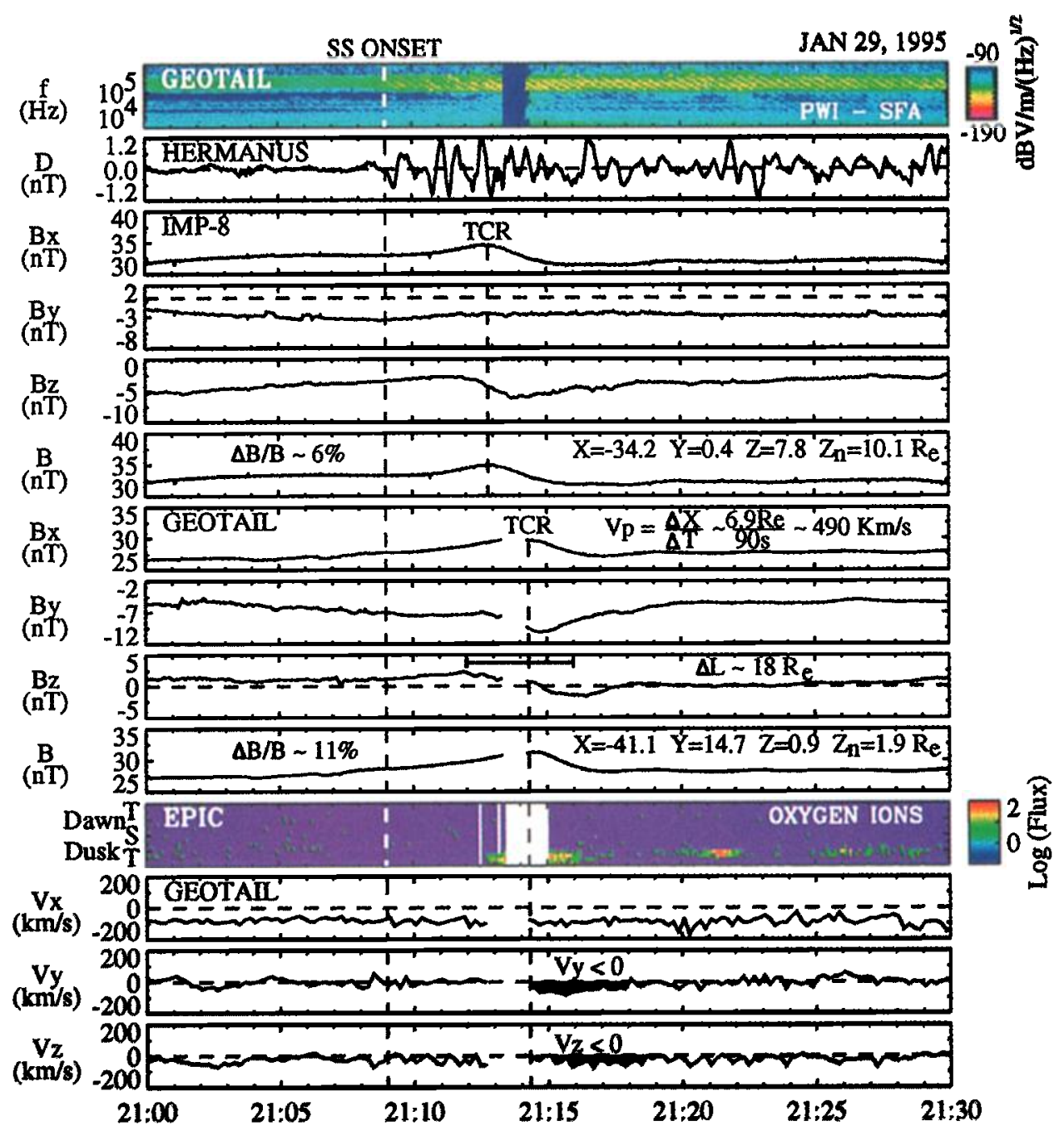

Figure 1. A compilation of measurements from IMP 8, Geotail and Hermanus Observatory for the TCR event on January $29,1995$.

The more distant Geotail, in contrast, measured a weak, but distinct, northward $\mathrm{B}_{\mathrm{z}}$ draping signature followed by a southward $B_{z}$ with the inflection point lying near the peak in the field magnitude. The duration of the TCR is about twice that seen by IMP 8 , i.e. $\sim 4 \mathrm{~min}$. The start of the decrease in the $B_{z}$ perturbation at Geotail commences very near the time that $B_{z}$ begins to become more southward at IMP 8, 21:12. The fact that this change in field draping is seen simultaneously at both s/c strongly suggests that they were both near the plasmoid at the time when it began to be ejected tailward with IMP 8 over the central region of the plasmoid bulge and Geotail farther tailward where it experienced more of the complete draping signature. However, the tailward movement of a plasmoid bulge with a constant shape and size would have resulted in the start of the decrease in $\mathrm{B}_{\mathrm{z}}$ at Geotail being delayed relative that observed at IMP 8. We attribute the lack of a delay in the onset of the decrease in $\mathrm{B}_{\mathrm{z}}$ to a continued rapid growth in the size of the plasmoid during and after its release as discussed below.

The peak field compression at Geotail comes $\sim 90 \mathrm{sec}$ following the peak at IMP 8. From this we can infer a downtail speed for the underlying plasmoid of

$$
\mathrm{V}_{\mathrm{pmd}} \sim \Delta \mathrm{X} / \Delta \mathrm{T} \sim 490 \mathrm{~km} / \mathrm{s}
$$

Combined with the 4 min TCR duration, measured from the $\pm B_{z}$ maxima, the inferred length of the plasmoid in the $X$ direction is

$$
\mathrm{L}_{\mathrm{x}} \sim \mathrm{V}_{\text {pmd }} \mathrm{x} \Delta \mathrm{T} \sim 18 \mathrm{R}_{\mathrm{e}}
$$

which is comparable to what was seen in the Geotail and ISEE 3 plasmoid surveys (leda et al., 1998; Moldwin and Hughes, 1992). If the near-Earth neutral line (NENL) producing this plasmoid was located near its average location of $X \sim-26 R_{e}$ (Nagai et al., 1998), then, at the time of substorm onset, the center of the plasmoid bulge would have been at $X \sim-26-$ $(18 / 2)=-35 R_{e}$ with its tailward end extended to $X \sim 44 R_{e}$. We present an idealized view of the location of both $\mathrm{s} / \mathrm{c}$ relative to the plasmoid in Figure 2.

\section{Geotail Energetic Particles}

The eleventh panel in Figure 1 shows the directional anisotropies of 186.5-221.4 $\mathrm{keV} \mathrm{O}^{+}$ions observed at Geotail. The channel shown represents the lowest energies normally sampled by the EPIC instrument for this species. These oxygen observations show a small, but significant enhancement above background levels during the TCR passage. Several inferences can be drawn from this observation. First, the enhancement in oxygen ions is confined to those particles moving in a tailward 


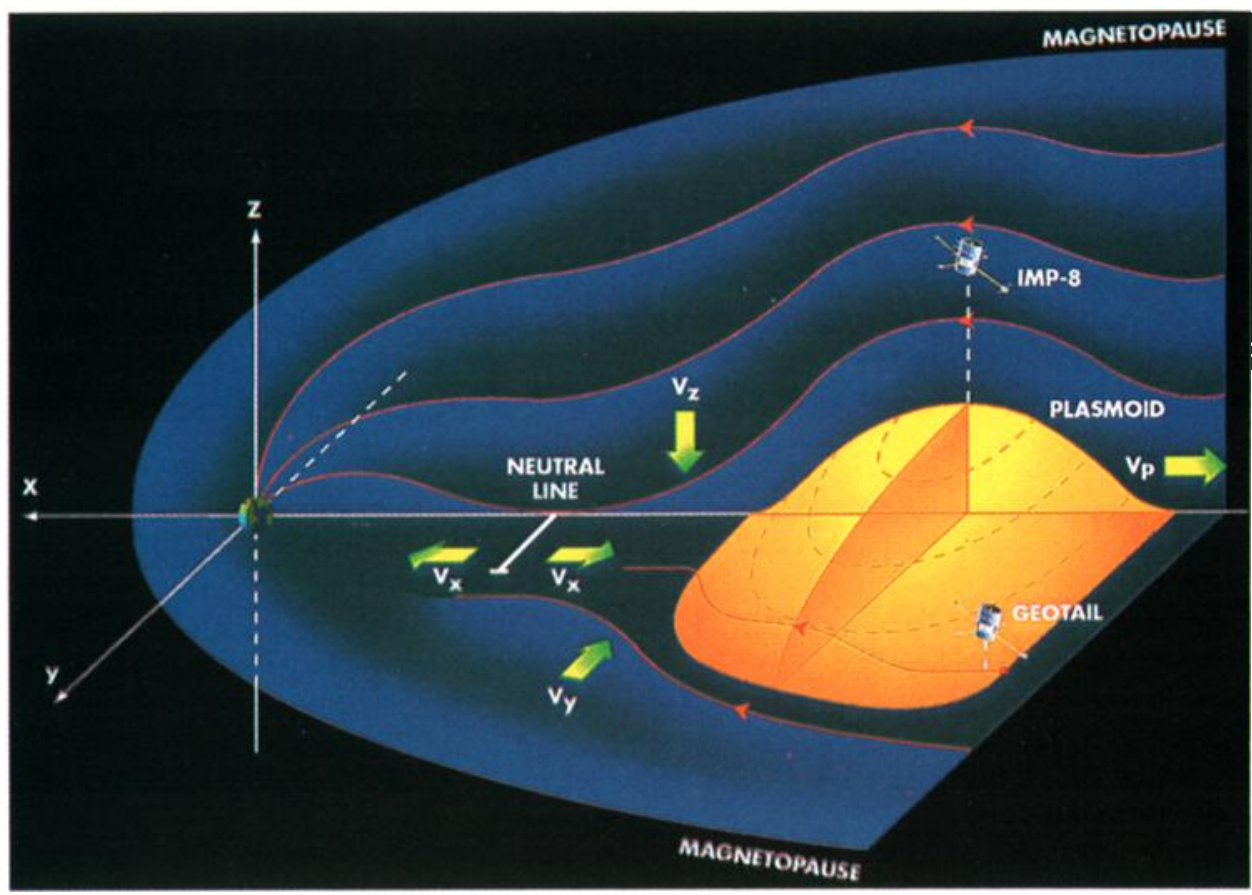

Figure 2. A schematic view of plasmoid formation and ejection relative to IMP 8 and Geotail on January 29, 1995.

and duskward direction. This is consistent with the dawn-todusk gradient anisotropy expected as the $s / c$ enters the plasma sheet boundary layer. The gyroradius of these oxygen ions in the $30 \mathrm{nT}$ field observed at the center of the TCR is $\sim 1.4 \mathrm{R}_{\mathrm{e}}$. The corresponding gyroradius for $67 \mathrm{keV}$ protons is about 0.2 $\mathrm{R}_{\mathrm{E}}$. However, no enhancement was observed in the fluxes of protons at this time suggesting that Geotail was between 0.2 and $1.4 \mathrm{R}_{\mathrm{e}}$ from the edge of the plasma sheet boundary layer as the plasmoid passed this spacecraft. This conclusion is also supported by the plasma beta values of $<0.01$ (not shown) calculated from the Geotail LEP plasma data.

A second inference can be made by looking at the directional dependences of the oxygen enhancement (not shown). As well as recording particle counts as a function of azimuthal sector as the spacecraft spins, the EPIC instrument has 2 telescopes, one of which looks at an angle of $23^{\circ}$ above the ecliptic, while the other points $23^{\circ}$ below the ecliptic. The oxygen enhancement in Figure 1 is seen only in the telescope/sector combinations viewing particles moving in a tailward, duskward and southward direction. Given the s/c location towards the dusk flank of the tail, this is consistent with the flux enhancement being located below Geotail and towards the tail center. Hence, it appears that the PSBL is pushed both northward and duskward over the spacecraft location at this time. Taken together, the EPIC ion fluxes and their anisotropies argue most strongly that this plasmoid was limited in its east-west extent and that Geotail was located very close, $\sim \pm 1 R_{e}$ to its duskward edge as depicted in Figure 2.

\section{Lobe Reconfiguration After Plasmoid Ejection}

Another very significant feature of these Jan 29, 1995 observations is the strong unipolar $B_{y}$ perturbation in the Geotail TCR measurements. The typical TCR $B_{y}$ perturbation, when present, is bipolar and smaller in amplitude than that observed in $B_{z}$ (Slavin et al., 1993). Unipolar $B_{y}$ pulses, such as that displayed in Figure 1 , indicate a shift in field line location toward the center of the tail which persists for some time following the passage of the plasmoid. Hence, the lobe magnetic flux tubes appear to be shifted into the "wake" region of the tailward moving plasmoid as shown in Figure 2 with the sample field line in red. If this interpretation, first suggested by Taguchi et al. (1998), is correct, then this flux tube transport should be accompanied by a dawnward flow in the Geotail plasma observations. Indeed, this is the case as shown in the bottom panels of Figure 1 where a $V_{y} \sim-50 \mathrm{~km} / \mathrm{s}$ flow commences nearly coincident with the unipolar $B_{y}$ pulse in the magnetic field data. $A$ weak negative, i.e. southward, $V_{z}$ flow toward the plasma sheet is also present. Hence, the Geotail measurements taken very close to the dusk flank of this plasmoid strongly suggest a reconfiguration of the lobe magnetic fields which transports open flux tubes into the wake region and downward toward the near-Earth neutral line where they may undergo reconnection.

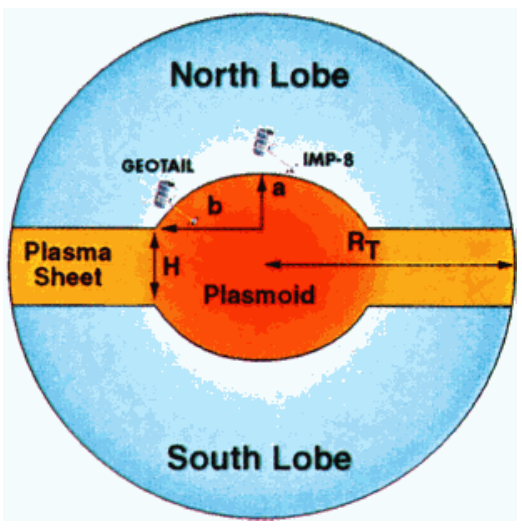

Figure 3. A cross-sectional view of the tail cutting the January 29,1995 plasmoid at the point of its maximum thickness. 


\section{Plasmoid Evolution After Release}

The recent Geotail plasmoid survey by leda et al. (1998) has produced strong statistical evidence for a rapid growth in plasmoid "width" (i.e., Y direction) and "length" (i.e., X direction) immediately following release. The dual $\mathrm{s} / \mathrm{c}$ observations of January 29, 1995 are also of great interest in regards to this issue. The reason is that in addition to providing measures of plasmoid length and width, our TCR observations can be used to infer the "height" of the plasmoid bulge in the $Z$ direction through modeling of the magnetic perturbation.

We propose to model the cross section of the plasmoid as half ellipses emerging from a slab plasma sheet with semiminor and semi-major axes of $a$ and $b$ directed in the $Z$ and $Y$ directions, respectively, as indicated in Fig. 3. In this manner, we can take into account the great decrease in plasmoid height observed in this study between $\sim 0$ (i.e., IMP 8) and $\mathrm{Y} \sim 15$ $R_{e}$ (i.e., Geotail). The total thickness of the plasma sheet and the radius of the tail are taken to be $\sim 7 R_{e}$ and $25 R_{e}$ (Fairfield, 1992). However, it should be noted that the tail still flares slightly at $X \sim-35$ to $-45 R_{e}$ and its radius is slowly increasing as evidenced by the $\sim 5 \mathrm{nT}$, or $17 \%$, reduction in lobe field strength between IMP 8 and Geotail in Figure 1. This corresponds to an $\sim 8 \%$ increase in tail radius in going from the downstream distance of IMP 8 to Geotail.

For either lobe region, conservation of magnetic flux allows one to solve for the semi-minor axis of the plasmoid bulge, which corresponds to its half-height relative to the plasma sheet

$$
a=1 / b\left(1-B_{L} / B_{C}\right) R_{T}^{2}-2 H / \pi b\left(1-B_{L} / B_{C}\right) R_{T}
$$

where the subscripts $\mathrm{L}$ and $\mathrm{C}$ refer to the undisturbed lobe field and the value at the point of maximum compression, respectively. We use $b=15 R_{e}$ based upon our Geotail observations of the duskward extent of the plasmoid bulge. Using $\mathrm{B}_{\mathrm{C}} / \mathrm{B}_{\mathrm{L}}=1.06$ and 1.11 from the IMP 8 and Geotail magnetic field data in Eq. (3) then yields $a=2$ and $3.4 R_{e}$, respectively, for the plasmoid bulge at IMP 8 and Geotail. These semi-minor axes correspond to total plasmoid northsouth extents, i.e., $\mathrm{H}+2 \mathrm{a}$, at their tallest points of $\sim 10.9$ and $13.8 \mathrm{R}_{\mathrm{e}}$, respectively. Hence, the increase in total plasmoid height during its transit from IMP 8 to Geotail is about $\sim 3 R_{e}$. Taking into account the small increase in tail diameter between the downtail distance of these two $\mathrm{s} / \mathrm{c}$ noted earlier increases our estimate of the increase in plasmoid height at Geotail relative to IMP 8 to $\sim 4 R_{\mathrm{e}}$.

The implication of these model calculations is that the plasmoid causing these TCR perturbations expanded $\sim 2 R_{e}$ in both the north and south directions during the $90 \mathrm{sec}$ separating the IMP 8 and Geotail encounters. This, in turn, corresponds to an expansion speed of

$$
V_{Z}=\Delta Z / \Delta T \sim 2 R_{e} / 90 \mathrm{sec} \sim 140 \mathrm{~km} / \mathrm{s}
$$

Such expansion speeds are comparable to the internal bulk flows reported by Ieda et al. (1998) within "young" plasmoids and attributed to a rapid plasmoid growth immediately following ejection. However, it must be noted that our calculation has assumed for simplicity that all plasmoid growth to have been in its "height" whereas it probably also included its "width" (e.g., Ieda et al., 1998). Hence, this estimate of rate of growth in the $Z$ direction could equally well apply to the $Y$ direction or some combination of the two. These magnetic flux conservation arguments are sensitive only to the increase in the total cross sectional area of the plasmoid bulge.

\section{Summary}

This study made use of the simultaneous measurements returned by IMP 8 and Geotail on January 29, 1995 before, during, and just following ejection of a plasmoid. We have shown an instance where dual spacecraft measurements can be used to model all three dimensions of the underlying plasmoid and to estimate of its rate of expansion. For this event plasmoid dimensions of $\Delta X \sim 18, \Delta Y \sim 30$, and $\Delta Z \sim 10 R_{e}$ were inferred from the IMP 8 and Geotail magnetic field measurements. The earthward end of the plasmoid was inferred to be near the mean location of the near-earth neutral line, $X \sim-26 R_{e}$. Its center was underneath IMP 8 at $X \sim-34 R_{e}$ and its tailward end appeared to be near $X \sim-44 R_{e}$ or just beyond Geotail. Modeled using conservation of magnetic flux, the increase in TCR amplitude between the IMP8 and Geotail encounters implies that the underlying plasmoid was expanding at a rate of $\sim 140$ $\mathrm{km} / \mathrm{s}$ or $1.3 \mathrm{Re} / \mathrm{min}$. Finally, the magnetic field and plasma flow velocity variations accompanying and following the TCR at Geotail indicate that a reconfiguration of the lobe magnetic field closely followed the ejection of the plasmoid which moved magnetic flux tubes into the wake behind the plasmoid where they would convect into the near-earth neutral line and release energy through reconnection to continue powering the substorm.

Acknowledgments. The authors gratefully acknowledge access to magnetic field data from the IMAGE Chain provided by the Finnish Meteorological Institute and energetic particle measurements from the Los Alamos National Laboratory. Discussions with A. Ieda regarding plasmoid formation also contributed significantly to this study.

\section{References}

Fairfield, D.H., On the structure of the distant magnetotail: ISEE 3, J. Geophys. Res., 97, 1, 403, 1992.

leda, A., et al., Statistical analysis of the plasmoid evolution with Geotail observations,J. Geophys. Res., 103, 4, 453, 1998.

Moldwin, M.B., and W.J. Hughes, On the formation and evolution of plasmoids: A survey of ISEE 3 data, J. Geophys. Res., 97, 19, 259, 1992.

Nagai, $T$., et al., Structure and dynamics of magnetic reconnection for substorm onsets with Geotail observations, J. Geophys. Res., 103, $4,419,1998$.

Slavin, J.A. et al., ISEE 3 observations of traveling compression regions in the magnetotail, J. Geophys. Res., 98, 15, 425, 1993.

Taguchi, S., et al., Temporal relationship between mid-tail TCRs and substorm onset: Evidence for near-Earth neutral line formation in the late growth phase, J. Geophys. Res., 103, 26, 607, 1998.

J.A. Slavin, M. Hesse, D.H. Fairchild, and R.P. Lepping NASA/GSFC, Greenbelt, MD 20771 (james.a.slavin@gsfc nasa gov)

C.J. Owen Lepping Queen Mary and Westfield College, Astronomy

Unit, Mile End Road, London E1 4NS, U.K. (c.j.owen@gmu.ac.uk)

S. Taguchi The University of Electro-Communications, Dept. of

Electronic Engineering, Chofu, Tokyo, 182 Japan (taguchi@ee.uec.ac.jp)

T. Mukai ISAS, 3-1-1 Yoshinodai, Sagamihara, Kanagawa 229-0022, Japan (mukai@stp.isas.ac.jp)

A.T.Y. Lui JHU/Applied Physics Laboratory, Laurel, MD 20723 (lui@merritt..huapl edu)

R.R. Anderson Department of Physics and Astronomy, University of Iowa, lowa City, IA 52242 (anderson@iowave.uiowa.edu)

H. Matsumoto Radio Atmospheric Science Center, Kyoto University,

Uji, Kyoto 611-0011, Japan (matsumot@kursac.kyoto-u.ac.jp)

P.R. Sutcliffe Hermanus Magnetic Observatory, P.O. Box 32,

Hermanus 7200, South Africa (psutclif@csir.co.za)

(Received June 4, 1999; accepted July 7, 1999) 